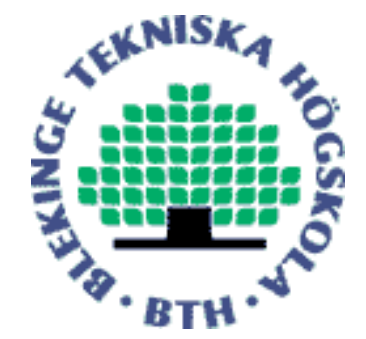

Copyright (C) 2012 IEEE.

Citation for the published paper:

On Routing in Cognitive Radio Networks

Alexandru Popescu, Markus Fiedler

9th International Conference on Communications (COMM2012)

2012 Bucharest

This material is posted here with permission of the IEEE. Such permission of the IEEE does not in any way imply IEEE endorsement of any of BTH's products or services Internal or personal use of this material is permitted. However, permission to reprint/republish this material for advertising or promotional purposes or for creating new collective works for resale or redistribution must be obtained from the IEEE by sending a blank email message to pubs-permissions@iee.org.

By choosing to view this document, you agree to all provisions of the copyright laws protecting it. 


\title{
On Routing in Cognitive Radio Networks
}

\author{
Alexandru Popescu ${ }^{1, *}$ and Markus Fiedler ${ }^{1}$ \\ ${ }^{1}$ Blekinge Institute of Technology, Dept. of Communications and Computer Systems, Karlskrona, Sweden \\ ${ }^{*}$ Corresponding author (E-mail : app@bth.se)
}

\begin{abstract}
Cognitive Radio Networks (CRNs) are expected to address and to resolve important technological and operational challenges of future networks, such as Dynamic Spectrum Access (DSA), routing in heterogeneous networks and provision of Quality of Experience (QoE) for different applications. Accordingly, a number of new terminal and network functionalities are required to solve the technical problems and to provide efficient management of CRNs. The paper advances a new solution for routing in CRNs, providing communication between Secondary Users (SUs) occupying different so-called Spectrum Opportunities (SOPs), i.e., portions of spectrum not occupied by Primary Users (PUs). This entails solving a complex process composed by two fundamental elements. These elements are multi-constraint routing and multi-dimensional adaptation for multiple cognitive radio dimensions like space, frequency, power and time. The goal of the paper is to develop optimization algorithms necessary to provide end-to-end (e2e) routing paths for communication between SUs with associated QoE demands.

Key words - Cognitive radio networks management; spectrum access; route optimization; quality-of-experience.
\end{abstract}

\section{INTRODUCTION}

Resource fluctuations, churn and PUs behavior represent challenging routing problems in CRNs. Another important parameter that must be considered is the risk of resource starvation for intermediate cognitive radio devices (CRDs). During which, CRDs may not be able to deserve QoS guarantees for e2e routes passing through them. Additionally, it is important to consider the applications, and their degree of tolerated quality degradation for brief time periods or recovery from more severe quality degradation by using Forward Error Correction (FEC) codes or retransmissions. This is today expressed in terms of the so-called QoE parameter.

Consequently, routing in CRNs is a very demanding problem similar to routing in overlay networks [7]. The complexity in this case is due to multiple constraints imposed on the $\mathrm{e} 2 \mathrm{e}$ routes, the presence of dynamic environments in terms of quick changes in the environment (PUs behavior, resource fluctuations and churn) as well as the demand for realtime performance in terms of adaptation to each link-state update. Basically, the $\mathrm{CRN}$ routing can be partitioned in three categories with reference to the activity and holding times of licensed users: Static, Dynamic and Opportunistic [7], [2].

A novel solution for routing in CRNs is advanced in our paper, where the holding times of the routes are considered to be down to an order of minutes (static/dynamic temporal framing). This entails solving a complex process composed by two fundamental elements, multi-constraint routing and multi-dimensional adaptation for multiple CR-dimensions like space, frequency, power and time. The rest of the paper is as follows. Section II is about multi-constraint routing in CRNs and the associated multi-dimensional adaptation. Section III is about the system model considered in our research. Section IV further details this model with a formal description. Section $\mathrm{V}$ is developing on multi-objective constraint optimization. Finally, section VI concludes the paper.

\section{Multi-Constraint Routing In CRNs}

There are three essential parts regarding the routing in CRNs. These are the definition of an appropriate QoS metric for the e2e routing, the path selection algorithm and the routing algorithm. A set of constraints are typically associated with the notion of QoS of an e2e route. This set may refer to link, path and tree constraints or combinations. An e2e route is composed by a number of links, where every link has some residual resources available, in the particular time period of a e2e communication. The challenge is to find out e2e paths to satisfy the e2e QoS constraints. To do this, local state information and/or global state information can be used. A particular difficulty is because an e2e route may involve crossing different IP domains as well as different spaces, e.g., geographical, frequency and power.

In general, the path selection problem is viewed as an optimization problem. A network is in this case represented by a directed graph function of a set of vertices (nodes) and a set of directed links (edges). Each link can be associated with a set of additive metrics (e.g., delay, cost) and/or concave metrics also known as min-max metrics (e.g., bandwidth, policy flags) and/or multiplicative metrics (e.g., packet loss). The measure of an e2e path can accordingly be either additive (for delay, or cost), or minimum of the metrics along the particular e $2 \mathrm{e}$ path (for bandwidth) or the product of the metrics along the e2e path (for packet loss). Problems involving constraints on concave metrics can easily be solved by pruning the links of the graph not satisfying the particular constraints. Multiplicative weights can be turned into additive weights by taking the logarithm of their product. On the other hand, additive metrics are more difficult to handle and demands solving sophisticated optimization problems. Finally, situations may arise when several goals must be simultaneously achieved (in doing e2e routing) such as the QoS/QoE requirements of the particular applications are met.

Examples of parameters subject to simultaneous optimization computations are throughput, e2e latency, cost and packet/bit error rate. A serious problem, is when objectives conflict with each other due to existent correlations among some of them. For instance, a conflictual situation may exist 
in the case of simultaneously trying to minimize the latency and the bit error rate (BER). The conflict occurs because the packet size affects these two parameters in an opposite way. Theoretically, in the case of multi-objective constraint optimization, the optimal solutions, for the above-mentioned particular example, lie in the so-called Pareto optimal front [3]. In a similar way, other theoretical models are suitable for other particular situations. Another serious challenge is because scheduling and routing algorithms may have different operation time scales. This in turn becomes a serious challenge to be solved for improving the protocol efficiency.

With reference to the suggested architecture in [2], the focus must be on path selection algorithms that can handle multiple constraints (especially linear constraints) and multiple objectives (e.g., simultaneous minimal delay and maximum bandwidth). Furthermore, the routing and multi-dimensional adaptation algorithms must provide e2e routes in geographical space regardless of the necessary adaptations needed in other $\mathrm{CR}$ dimensions like frequency and power. By routing we mean the determination process of an e2e route. Whereas by multidimensional adaptation we mean the adaptation process of a SUs operating parameters to the constraints of available SOPs in terms of frequency, power, geographical point and time.

Another important criteria for the design of optimal routing solutions is the avoidance or reduction of interference among different routes, with the positive effect of improving the overall network performance. The e2e routing algorithms are demanded in our case to be able to cope with dynamic environments in form of, e.g., resource fluctuations and churn. Finally, a further complication is because such algorithms have rather hard "real-time" performance demands, which means, they are requested to quickly compute new feasible routes for each link-state update.

\section{SySTEM MODEL}

We assume a decentralized sensing-based scheme for cognitive medium access. The sensing information is collected by both CRDs and a Support Node (SN), which typically resides in the Base Station (BS). SN compiles the e2e routing path for a particular communication between two CRDs [2]. Where, intermediate CRDs along the e2e path may need to update the communication path, in order to adapt to dynamic processes in the local radio environment during the communication process. For instance, a PU may take over a particular SOP, which was part of the e2e routing path. To maintain the operating parameters for different CR-dimensions a multi-dimensional data-representation is used at the SN [2]. Depicting the current network state together with available SOPs and operational parameters of network members makes it possible to balance the traffic in the CRN and optimize routing paths according to user constraints.

Hence, at a particular time moment $t_{j}$, we have information about the availability of a number of SOPs, which are denominated by $\left\{S O P_{i}\left(t_{j}\right)\right\}_{i=1-n}$. A SOP can be defined as a function of multiple metrics as $S O P=$ $f$ (bandwidth, expectationtime, power, interferencelevel, geographicpoint,...). Every $S O P$ is described in a fourdimensional space in terms of geographical point (referred

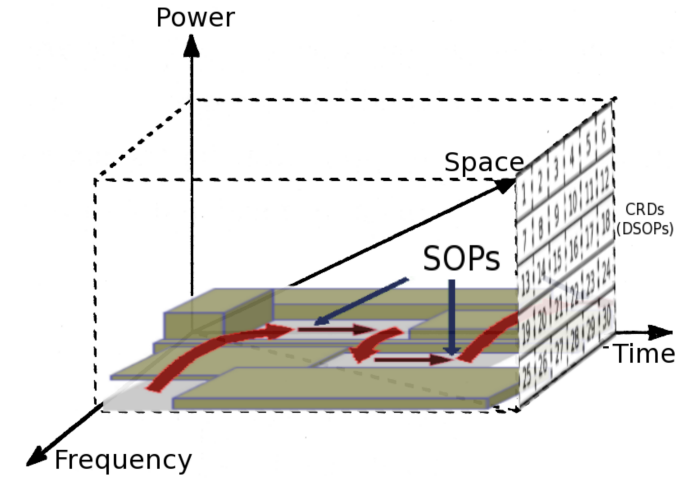

Figure 1. Example of adaptations in a multi-dimensional CR space

to as space in the continuation), power, frequency and time. We ignore at the moment other metrics like, e.g., interference level. Additionally, information about the expected temporal availability of these $S O P s$ is also assumed, which can be obtained based on traffic measurements and analysis as well as prediction methods like the ones suggested in [1].

Based on this information, the SN decision making entity must compute an e2e route from a SU sender to a SU receiver, which may traverse different SOPs. This is a multi-step process that depends on:

Data Collection: from network elements, which is required for CRDs to be properly adapted to the available SOPs. Based on the collected data, a composite e2e routing metric can be used between CRDs in a particular CRN. The data is collected through spectrum sensing by both the $\mathrm{SN}$ itself (on a global geographical area for the CRN) and the CRN members;

Multi-Dimensional CRD adaptation: which is the process of constraining CRDs to the available SOPs in terms of frequency, power, space and time. The multi-dimensional adaptations allow CRDs to operate in the SOPs available in the area of a particular CRN, as shown for example in figure 1

The conditions for adaptations are characterized by the set of frequency $F_{d}$, power $P_{d}$, space $S_{d}$ and time $t_{d}$ like:

$$
\begin{gathered}
\left\{F_{\text {min }} \leq F_{d} \leq F_{\max }\right\} \cap\left\{P_{d} \leq P_{\max }\right\} \cap\left\{S_{d} \equiv S_{\text {set }}\right\} \cap\left\{t_{\text {start }} \leq\right. \\
\left.\Delta_{d} \leq t_{\text {end }}\right\}
\end{gathered}
$$

where $F_{\min }$ and $F_{\max }$ represent the minimum and maximum allowed frequencies for the operation of a particular CRD $d$. Since a large available SOP may accommodate the operation of several CRDs by dividing it into several channels/subcarriers (the total depends on the employed coding modulation). In the continuation, this will be referred to as a Device Spectrum Opportunity (DSOP), i.e., each DSOP implies one CRD operation. $P_{\max }$ represents the maximum allowed power output for CR device $d . \quad S_{d}$ are the space coordinates for the SOP where CRD $d$ is set to operate. This can for instance be any type of localization coordinates that depict the geographical point of a particular CRD operation zone. $t_{\text {start }}$ is the starting time moment when the particular SOP is available and $t_{\text {end }}$ is the time moment when a particular SOPs availability expires, meaning $\Delta_{d}$ is the time interval during which the particular SOP is available for SU operation;

Route Computation and Optimization: the data collected 
from the network elements allows us to compute an e2e route from a particular source to a particular destination in the $\mathrm{CRN}$, which may require several intermediate devices to be traversed along the path. Devices in the CRN are seen as different DSOPs given their operation within the constraints of individual SOPs (from the pool of all SOPs available for SU operation). Thus, finding a route $r$ means setting up a communication path between the different DSOPs that are used in the particular CRN, i.e., a source $D S O P_{x}$ and a destination $D S O P_{y}$, which uses a number $z$ of available $D S O P s$, e.g., $D S O P_{x}-D S O P_{1}-D S O P_{2}-\ldots D S O P_{z}-D S O P_{y}$. Every $D S O P$ in the $\mathrm{CRN}$ is characterized by the parameters mentioned above (power, frequency, space and time) and the associated statistics, which are measured and determined offline, under a long time period. This information is recorded in a so-called Historical Statistics Database (HS-DB) [2]. This process can further be viewed as a Multi-Constrained Path (MCP) optimization problem where the path conditions are characterized by throughput $T H_{r}$, cost $C_{r}$, time interval $t_{r}$ and delay $\Delta_{r}$ :

$$
\left\{T H_{\min } \leq T H_{r}\right\} \cap\left\{C_{r} \leq C_{\max }\right\} \cap\left\{t_{\min } \leq t_{r} \leq t_{\max }\right\} \cap\left\{\Delta_{r}\right\}
$$

where $T H_{\min }$ is the minimum required throughput for the e2e route, $C_{\max }$ is the maximum accepted cost for the route, $t_{\min }$ and $t_{\max }$ are the starting and ending time moments within which a route with the requested resources is needed i.e., only routes that become available in the time interval $t_{r}$ are considered. $\Delta_{r}$ is the minimum expected time of all expected times associated with the selected DSOPs, i.e., $D S O P_{1}, D S O P_{2}, \ldots D S O P_{z}$. Every hop along the path is seen as a different $D S O P$ and the time it takes to traverse them all is considered to be $\Delta_{r}$, i.e., expected path delay.

It is assumed that the DSOPs belonging to the selected route $r$ are available long enough to allow for the route to be set up. To achieve this, statistics collected over a long time period, different prediction models, e.g., Moving Average (MA), Auto Regressive (AR), Autoregressive moving average model (ARMA) and suitable decision making process based on solutions like in [1] are used.

\section{FORMAL DESCRIPTION}

The formal description of the multi-dimensional CRD adaptation process, where the operation of a particular CRD is constrained to the available parameters of a given DSOP, is:

\footnotetext{
Given

a pool of $k$ sensed SOPs at a particular time moment $t_{j}$, which are denominated by $S O P_{i}\left(t_{j}\right)_{i=1-n}$

Adapt the parameters of CRD $d$ to operate in DSOP $n$ from the pool of available SOPs, denominated by $S O P_{k}$

Subject to $\quad\left\{F_{\min } \leq F_{d} \leq F_{\max }\right\} \cap\left\{P_{d} \leq P_{\max }\right\}$ $\cap\left\{S_{d} \equiv S_{\text {set }}\right\} \cap\left\{t_{\text {start }} \leq \Delta_{d} \leq t_{\text {end }}\right\}$
}

Whereas, the formal description of the process to find a route $r$ between $D S O P_{x}$ and $D S O P_{y}$ is:
Given
a pool of $n$ available devices, defined as DSOPs at a particular time moment $t_{j}$, which are denominated by $\operatorname{DSOP}_{i}\left(t_{j}\right)_{i=1-n}$
Find the route $r$ between two DSOPs (devices) in the pool (network), denominated by $D S O P_{x}$ and $D S O P_{y}$
Subject to

Depending upon the existent conditions at a specific time, several routes may be found between $D S O P_{x}$ and $D S O P_{y}$, for the expected temporal framing of the route. This means that one particular route will be selected among a number of available routes $1,2, \ldots w$, where all of them satisfy the general conditions characterized by throughput $T H_{r}$, cost $C_{r}$, time interval $t_{r}$ and delay $\Delta_{r}$. Consequently, selecting a single route requires an exact fitting of one of the path characteristics to the user requested QoE. Hence, this can be viewed as a Multi-Constrained Optimal Path (MCOP) optimization problem. To select one path from a set of feasible paths, it is desirable to minimize or maximize over one of the metrics or augment the initial path characterization with additional e2e goals like length $L_{r}$ (i.e., the length of the path should be minimized) or security level $S_{\min }$ (i.e., route $r$ should satisfy a minimum level of security). The formal description process of selecting one path among several is:

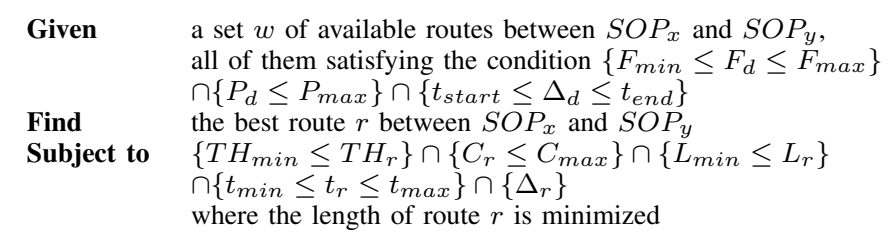

\section{Multi-Objective Constraint Optimization}

The selection of e2e routing paths subject to multiple constraints is defined as an optimization problem. This can be of type MCP optimization problem or MCOP optimization problem [4]. Several popular routing algorithms are Self-Adaptive Multiple Constraints Routing Algorithms (SAMCRA) [4] and Particle Swarm Optimization [5].

To solve optimization problems of type MCP and MCOP, several initial assumptions are considered [4]. A network is represented by a directed graph $\mathcal{G}=(\mathcal{N}, \mathcal{E})$, where $\mathcal{N}$ is a set of vertices (nodes) and $\mathcal{E}$ is a set of directed links (edges). Each link is associated with a set of additive QoS metrics and non-additive QoS metrics. We assume the number of QoS metrics is denoted by $m$. Each link can therefore be characterized by a $m$-dimensional link weight vector, which consists of $m$ non-negative QoS weights as components. Accordingly, the QoS measure of an e2e path can be either additive (in the case of, e.g., delay, cost) or minimum of the QoS weights along the particular e2e path (in the case of, e.g., bandwidth). Furthermore, we assume that, in addition to information about graphs and link weights, information about the flow demands is available as well. A flow demand is defined to be a set of path constraints for the path $P(s, d)$, where $s \in \mathcal{N}$ is the source node and $d \in \mathcal{N}$ is the destination node. The formal description of the optimization problems is as follows.

\section{A. Multi-Constrained Path (MCP) Problem}

For $i=1, \ldots, m$, we denote by $w_{i}(u, v)$ the $i$-th additive metric for the link $(u, v)$ between nodes $u$ and $v$ such as $(u, v)$ $\epsilon \mathcal{E}$. The MCP optimization problem for $m$ additive constraint values $L_{i}$ on the requested path can be formally described as: 
Given

a network $\mathcal{G}=(\mathcal{N}, \mathcal{E})$, where each link $(u, v) \in \mathcal{E}$ between nodes $u$ and $v$ is specified by a link-weight vector of $m$ additive QoS weights $w_{i}(u, v) \geq 0$, with $i=1,2, \ldots m$

Find the path $P$ from a source $s$ to a destination $d$

Subject to $\quad w_{i}(P)=\sum_{(u, v) \epsilon P} w_{i}(u, v) \leq L_{i}$ for $i=1, \ldots, m$

A path $P$ that satisfies all $m$ constraints is referred to as a feasible path. This means that the additive QoS measures for the weight of the path $P$ between nodes $s$ and $d$ equals the vector-sum of the weights of the constituent links. There may be multiple paths in the graph $\mathcal{G}=(\mathcal{N}, \mathcal{E})$ found to satisfy the constraints. Any of these paths is in this case considered to be a solution to the MCP problem. However, it might be desirable to select the path with smallest length from the set of feasible paths. The MCP optimization becomes in this case the MCOP optimization problem [4].

\section{B. Multi-Constrained Optimal Path (MCOP) Problem}

The MCP problem can be converted into a MCOP problem by minimizing or maximizing over one of the metrics $w_{i}$. In the case of minimizing the metric, the goal is to retrieve the path with the smallest length $l(P)$ from the set of feasible paths. The MCOP problem can in this case be formally described as:

\section{Given a network $\mathcal{G}=(\mathcal{N}, \mathcal{E})$, where each link $(u, v) \in \mathcal{E}$ between nodes $u$ and $v$ is specified by a link-weight vector of $m$ additive QoS weights $w_{i}(u, v) \geq 0$, with $i=1,2, \ldots m$ Find the path $P$ from a source $s$ to a destination $d$ \\ Subject to $\quad$ (i) $w_{i}(P)=\sum_{(u, v) \epsilon P} w_{i}(u, v) \leq L_{i}$ for $i=1, . ., m$ and (ii) $l(P) \leq l\left(P^{*}\right)$ for all $P^{*}$ between $s$ and $d$, and $P$ satisfying (i)}

$l(P)$ can be any function of the weights $w_{i}(P)$, for $i=$ $1, \ldots, m$. This is also known as the path-weight function over all metrics. One can therefore state that, minimizing a properly chosen length function, may result in an efficient use of the network resources as well as a reduction of the monetary cost.

\section{Implementation Aspects}

The optimal solutions for finding a route subject to constraints on two or more additive and multiplicative metrics have been mathematically proven to be NP-complete [6]. This means that the time required to exactly solve the $\mathrm{MC}(\mathrm{O}) \mathrm{P}$ problem can not, in the worst case, be upper-bounded by a polynomial function. The consequence is that heuristics or approximation algorithms must be used, which have a better chance of running in polynomial time. The main approaches used to solve problems of type MCP are Bandwidth Restricted Path (BRP), Restricted Shortest Path (RSP) and Metrics Combination (MC), each of them with own advantages and disadvantages [4]. A good example of appropriate heuristics is as follows. Four fundamental concepts have been showed are needed to be considered in a multi-constrained exact routing algorithm. These are [4]:

- Nonlinear measure for the path length $l(P)=$ $\max _{i=1, \ldots, m}\left(\frac{w_{i}(P)}{L_{i}}\right)$

- Need to compute k-shortest paths

- The principle of path nondominance, and
- The look-ahead concept

These concepts have been incorporated in SAMCRA, a self-adaptive multiple constraints routing algorithm. It has been showed that SAMCRA-like algorithms perform best at an acceptable computational cost [4]. Other interesting constraint-based path selection algorithms are Chen's approximate algorithms, randomized algorithms [4].

However, an important parameter that must be considered is regarding the expected QoE for end user. With reference to this, the solution suggested in our case is:

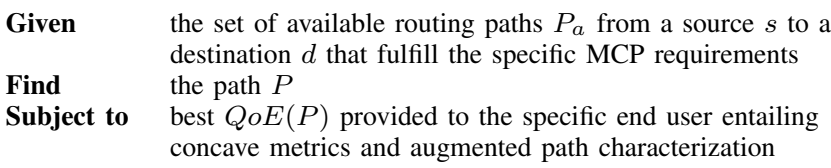

\section{CONCLUSIONS}

The paper has suggested a novel solution for routing in cognitive radio networks. This solution is part of a novel architecture for cognitive radio networks in beyond $4 \mathrm{G}$ networks, conceptualized for integration into existing base station solutions. Routing between secondary users occupying different spectrum opportunities demands for solving of multi-objective constraint optimization problems, which simultaneously considers the Quality of Experience for the individual users. The focus of the paper has been on research and implementation challanges. Future work is about developing mathematical and simulation models as well as performance evaluation.

\section{REFERENCES}

[1] Yao Y., Ngoga S.R., Erman D. and Popescu P.A., Competition-Based Channel Selection for Cognitive Radio Networks, IEEE Wireless Communications and Networking Conference, Paris, France, 2012

[2] Popescu O.A., Cognitive Radio Networks: Elements and Architectures, $\mathrm{PhD}$ thesis, in preparation, School of Computing, Bleking Institute of Technology, Karlskrona, Sweden, 2012

[3] Petrova M. and Mähönen P., Cognitive Resource Manager A Cross-Layer Architecture for Implementing Cognitive Radio Networks, chapter in book Cognitive Wireless Networks, Springer 2007

[4] Van Meghiem P. and Kuipers F.A., Concepts of Exact QoS Routing Algorithms, IEEE/ACM Transactions on Networking, 12(5), 2004

[5] Engelbrecht A.P., Fundamentals of Computational Swarm Intelligence, John Wiley \& Sons, 2006

[6] Wang Z. and Crowcroft J., Quality-of-Service Routing for Supporting Multimedia Applications, IEEE Journal of Selected Areas in Communications, 14(7), 1996

[7] Ilie D., and Popescu P.A., A Framework for Overlay QoS Routing, 4th Euro-FGI Workshop on "New Trends in Modelling, Quantitative Methods and Measurements" (WP IA.7.1), Ghent, Belgium, May/June 2007 